\title{
Conexión Venosa Pulmonar Anómala. Reporte de un Caso
}

\author{
Anomalous Pulmonary Venous Connection. A Case Report \\ Emilio Farfán C. ${ }^{1}$; Mark Echeverría M. ${ }^{1}$; Guillermo Salgado A. ${ }^{1}$; \\ Sebastián Navarro A. ${ }^{2}$; Verónica Inostroza R. ${ }^{1}$ \& Oscar Inzunza H. ${ }^{1}$
}

FARFÁn, C. E.; ECHEVERríA, M. M.; SALgAdO, A. G.; NAVARRO, A. S.; INOSTROZA, R. V. \& INZUNZA, H. O. Conexión venosa pulmonar anómala. Reporte de un caso. Int. J. Morphol., 36(4):1197-1201, 2018.

RESUMEN: Las conexiones anómalas de venas pulmonares fueron descritas por primera vez en 1739. Las anomalías de drenaje de las venas pulmonares se deben a una alteración temprana (día 27-30 del desarrollo) momento en el cual los plexos venosos pulmonares están en conexión con las venas de los sistemas cardinal, umbilical y vitelino, estas conexiones involucionan y el drenaje venoso deriva hacia el atrio izquierdo a través de la vena pulmonar común. En este trabajo presentamos una conexión venosa pulmonar anómala parcial, su conocimiento es relevante pues se asocia en un $80 \%$ a defectos cardiacos, principalmente comunicaciones interatriales. Disección de rutina de un cadáver adulto, de sexo masculino, a nivel del pedículo pulmonar izquierdo. Se describe una vena originada en el lobo pulmonar superior izquierdo que abandona el hilio pulmonar por delante del bronquio, realizando un trayecto ascendente de 10 $\mathrm{cm}$ de longitud desde el hilio para terminar desembocando en la vena braquiocefálica izquierda. La vena anómala drena el territorio del lobo superior del pulmón izquierdo. Por su disposición y relaciones, esta vena de trayecto anómalo tiene su origen en la persistencia de las conexiones del sistema venoso pulmonar, en especial las que se establecen con el sistema cardinal anterior izquierdo. Este hallazgo destaca la riqueza y relevancia clínica de la disección en la formación de profesionales de la salud.

PALABRAS CLAVE: Conexión Venosa; Vena pulmonar anómala; Variación anatómica.

\section{INTRODUCCIÓN}

El desarrollo del sistema venoso durante la quinta semana de vida intrauterina determina la formación de 3 sistemas principales, las venas vitelinas que llevan sangre del saco vitelino al seno venoso, las venas umbilicales que transportan desde las vellosidades coriónicas sangre oxigenada al embrión, y las venas cardinales que drenan el cuerpo del embrión (Sadler, 2014; Schoenwolf et al., 2009). Durante el desarrollo de estos sistemas pueden ocurrir alteraciones en los procesos naturales de evolución e involución venosa como los que afectan a las venas pulmonares como se describen en este caso.

Las conexiones anómalas de venas pulmonares cuya incidencia es de 2/100.000 nacimientos, corresponden a una cardiopatía congénita en que todas o algunas venas pulmonares drenan en el atrio derecho o en una vena sistémica en lugar de hacerlo en el atrio izquierdo, mezclándose la sangre oxigenada de las venas pulmonares con la sangre desoxigenada de las venas sistémicas. Lo más frecuente es que sólo una vena pulmonar presente una situación anómala; sin embargo, más de una vena puede tener drenaje anormal, siendo muy raro que involucre a todas ellas (Magalhães et al., 2016). Las conexiones venosas pulmonares anómalas de carácter parcial fueron descritas por primera vez en 1739 por Winslow; Wilson, en 1798, las describió en su forma total. El conocimiento embriológico del desarrollo venoso pulmonar es de gran utilidad para comprender la estructura de esta cardiopatía así como el origen de sus diferentes tipos de presentación total y parcial (Muñoz Castellanos et al., 2008).

Las anomalías de drenaje de las venas pulmonares se deben a una alteración temprana del desarrollo (día 2730 ), momento en el cual los plexos venosos pulmonares están en conexión con las venas de los sistemas cardinal y umbilicovitelino. Entre los días embrionarios 32-37, estas conexiones involucionan y el drenaje venoso de los pulmones deriva hacia el atrio izquierdo a través de la vena pulmonar común. En el caso de que estas conexiones en lugar de desaparecer se mantengan, las venas pulmonares izquierdas desembocarán en la vena braquiocefálica izquierda y las venas pulmonares derechas lo harán en la vena cava

${ }^{1}$ Departamento de Anatomía, Escuela de Medicina, Pontificia Universidad Católica de Chile, Santiago, Chile.

${ }^{2}$ Servicio de Urgencia, Hospital San Pablo de Coquimbo, Chile. 
superior (Orts Llorca, 1972); esto explica porque pueden ocurrir las anomalías parciales, es decir, afectando a un solo pulmón o lobo pulmonar. Una de estas conexiones transitorias es hacia la vena cardinal anterior izquierda, desde la cual surgen la vena cava superior izquierda y la vena vertical. En las conexiones venosas anómalas de tipo total, todas las venas pulmonares vierten su sangre a la circulación sistémica; en el caso de las conexiones venosas anómalas de tipo parcial, se incluyen desde 1 a 3 venas pulmonares que drenan su sangre a un colector que puede desembocar en el sistema de las venas cavas o en el atrio derecho (Hijii et al., 1998).

En este trabajo presentamos una conexión venosa pulmonar anómala de carácter parcial, que involucra a la vena pulmonar izquierda superior, correspondiente a una de las variedades menos frecuentes en este tipo de anomalías según el estudio de Hijii et al.; el conocimiento de esta anomalía es relevante pues se asocia a defectos cardiacos, principalmente comunicaciones interatriales.

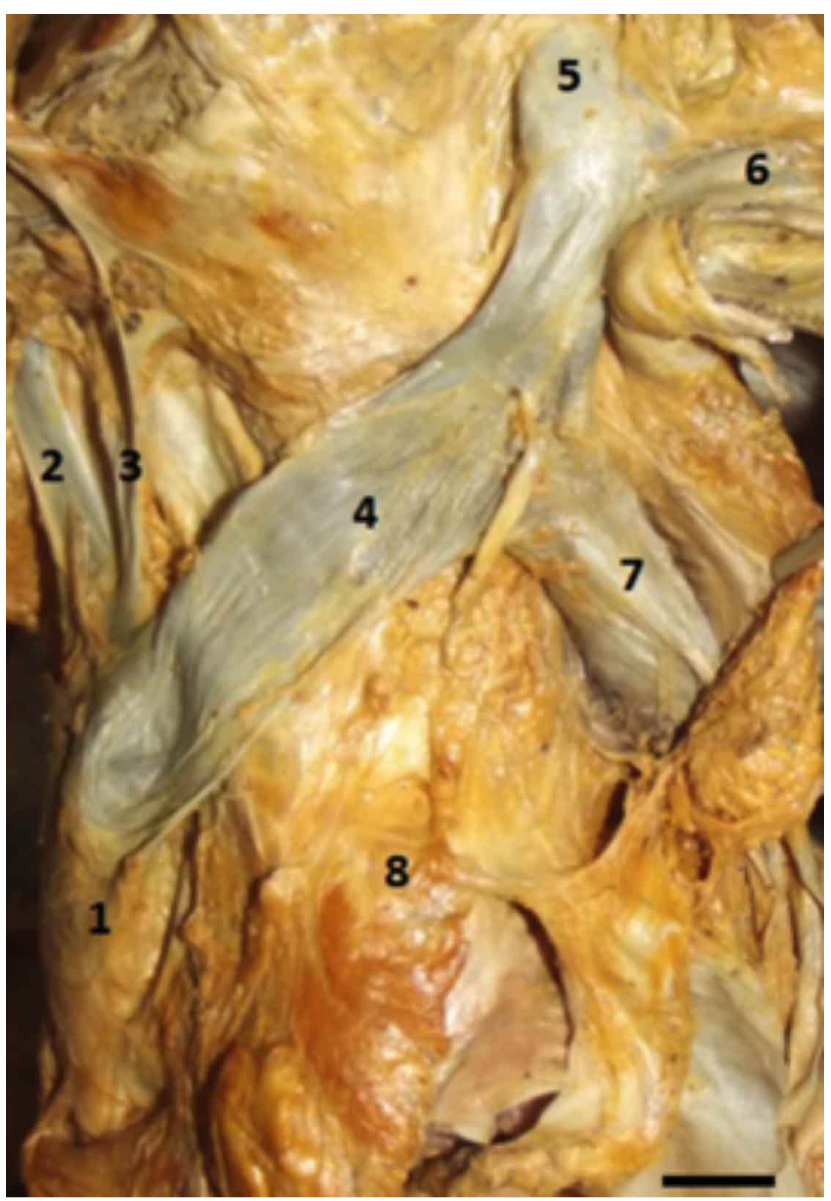

Fig. 1. Visión anterior del mediastino superior. 1. Vena cava superior; 2. V. Braquiocefálica derecha; 3. V. Tiroidea inferior; 4. V. Braquiocefálica izquierda; 5. V. yugular interna izquierda; 6 . V. Subclavia izquierda; 7. V. Pulmonar Superior Izquierda (VPSI); 8. Arco aórtico cubierto por pericardio. Barra negra $=1 \mathrm{~cm}$.

\section{MATERIAL Y MÉTODO}

Disección de rutina en una actividad práctica docente realizada en un cadáver de sexo masculino de 45 años de edad y cuya causa de muerte fue neumopatía aguda, procedimiento realizado en el Departamento de Anatomía Normal de la Escuela de Medicina de la Pontificia Universidad Católica de Chile.

Se realizó la disección de la piel y musculatura pectoral hasta exponer ambas clavículas, a continuación se practicó costotomía lateral bilateral, desde la primera hasta la octava costillas, siguiendo la línea axilar media hasta las líneas costales de la reflexión pleural. Se levantó el peto esternocostal y se separó la pleural parietal mediastínica del pericardio fibroso, se seccionaron ambos pedículos pulmonares; se retiraron los pulmones quedando intactos el pericardio fibroso y el corazón. A continuación, se realizó un corte longitudinal en la cara esternocostal del pericardio fibroso dejando expuesto el corazón. Para extraer este último se procedió a seccionar las venas cavas superior e inferior, la aorta ascendente, el tronco pulmonar y las arterias y venas pulmonares.

\section{RESULTADOS}

En la disección se detectó una vena de trayecto anómalo en el mediastino superior que desembocaba en la vena braquiocefálica izquierda, y que caudalmente resultaba de la confluencia de una vena común para las 2 venas bronquiales izquierdas en relación con el esbozo de una vena que surge en "fondo de saco" desde el pericardio fibroso que se relacionaba con la cara posterior del atrio izquierdo. A $1 \mathrm{~cm}$ de su conformación esta vena recibía a la vena pulmonar superior izquierda (VPSI) proveniente del lobo pulmonar correspondiente, lo que aumenta considerablemente su calibre. Cabe señalar que la VPSI abandonaba el hilio por delante del bronquio principal del mismo lado. La anatomía del pulmón izquierdo se encontraba normal y al igual que los elementos constituyentes del pedículo pulmonar.

La VPSI presentaba un trayecto ascendente de 8,5 $\mathrm{cm}$ hasta la vena braquiocefálica izquierda (VBCI), su dirección en el mediastino superior era de caudal a craneal y de izquierda a derecha, aumentando su calibre a medida que ascendía, (12 mm en su tercio inferior, $14 \mathrm{~mm}$ en su tercio medio y $17 \mathrm{~mm}$ en su tercio superior) (Tabla I). De este modo se formaba un tronco venoso que se ubicaba sobre el arco aórtico, en contacto con el saco pericárdico (Fig. 1). El vaso establecía relaciones anatómicas hacia posterior y medial con el ner- 
vio vago izquierdo y con los ramos cardiacos torácicos que procedían de él. Hacia anterior y lateral se relacionaba con el nervio frénico y con los vasos pericardiofrénicos de la antímera izquierda. La vena anómala recibía, en orden ascendente, el flujo sanguíneo del tronco común de las 2 venas bronquiales izquierdas, de la vena pulmonar superior izquierda, la vena hemiácigos accesoria (venas intercostales posteriores izquierdas 3,4 y 5), la vena pericardiofrénica izquierda, y pequeñas ramas pericárdicas y mediastínicas.

$\mathrm{Al}$ extraer el corazón y dejar el pericardio expuesto se pudo apreciar en su superficie interna los ostios de las venas pulmonares, siendo posible observar el cambio posicional que tuvieron las venas del lado izquierdo, de modo que la vena pulmonar inferior izquierda se disponía en el lugar de la vena superior, quedando el esbozo de la vena pulmonar superior izquierda en una posición superior, sin lumen ni permeabilidad, cubierta por pericardio seroso parietal.

Tabla I. Medidas de la vena pulmonar superior izquierda.

\begin{tabular}{lc}
\hline V.P.S.I. & \\
\hline Longitud & $8,5 \mathrm{~cm}$ \\
Calibre $1 / 3$ superior & $17 \mathrm{~mm}$ \\
Calibre $1 / 3$ medio & $14 \mathrm{~mm}$ \\
Calibre $1 / 3$ inferior & $12 \mathrm{~mm}$ \\
Venas Afluentes & 6 \\
\hline
\end{tabular}

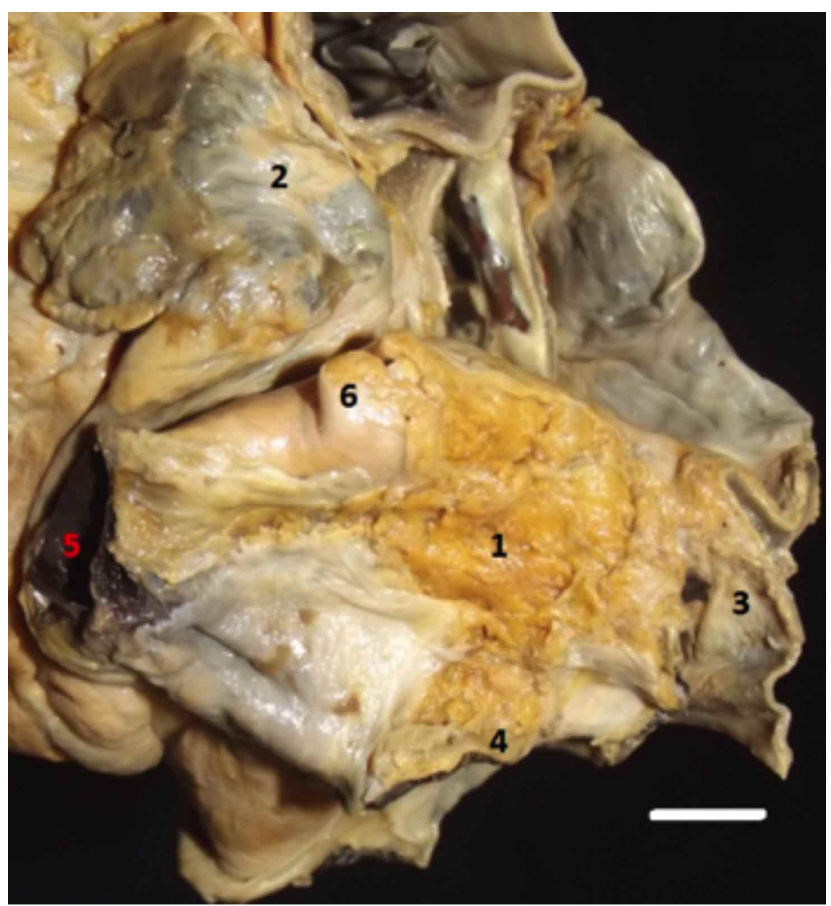

Fig. 2. Visión lateral izquierda de la base del corazón. 1. Atrio Izquierdo; 2. Aurícula Izquierda; 3. V. Pulmonar superior derecha; 4. V. Pulmonar inferior derecha; 5. V. Pulmonar inferior izquierda; 6. V. Pulmonar superior izquierda no permeable. Barra blanca $=1 \mathrm{~cm}$.
Observando el corazón desde su base se visualizó sobre la pared externa del atrio izquierdo el esbozo de la VPSI obliterada cubierta por pericardio seroso visceral, el cual no presentaba lumen ni era permeable (Fig. 2), en tanto que la superficie interna del atrio, a nivel de este mismo esbozo se era lisa. En el septo interatrial se evidenció un ostio que comunicaba ambos atrios, situación que se apreció mejor desde el atrio derecho. Este defecto septal que presentaba un área de $5 \mathrm{~mm}^{2}$, se ubicaba en la parte alta de la fosa oval, bajo el limbo, comunicando ambos atrios (Fig. 3).

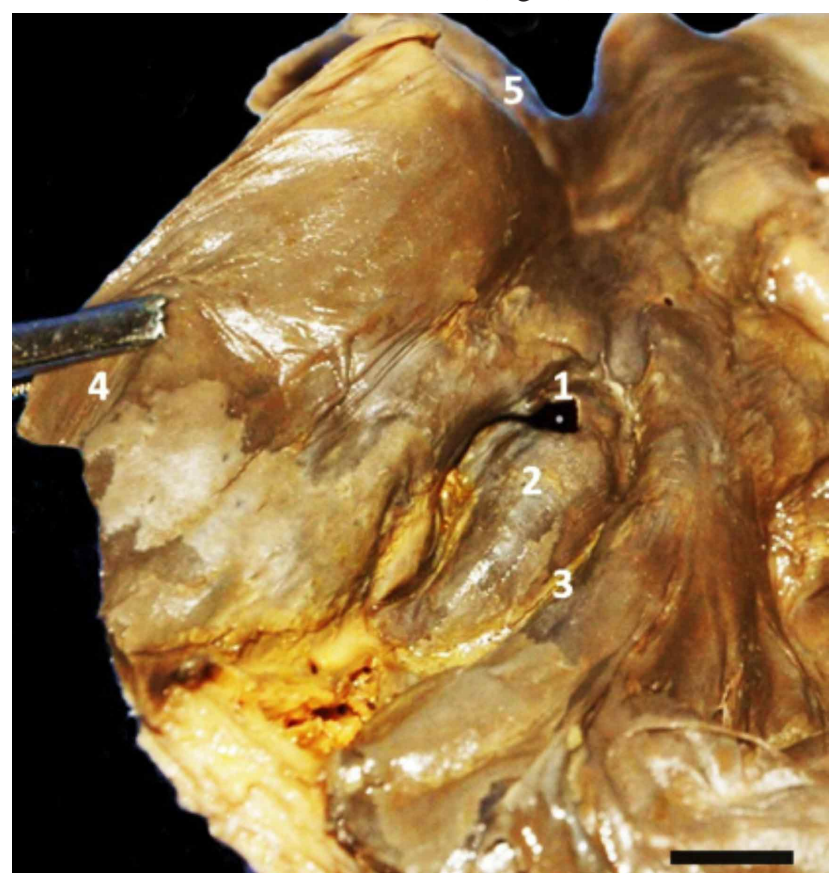

Fig. 3. Visión interna del atrio derecho. 1. Orificio interatrial; 2. Fosa oval; 3. Limbo de la fosa oval; 4. V. Cava inferior; 5. V. Cava superior. Barra negra $=1 \mathrm{~cm}$.

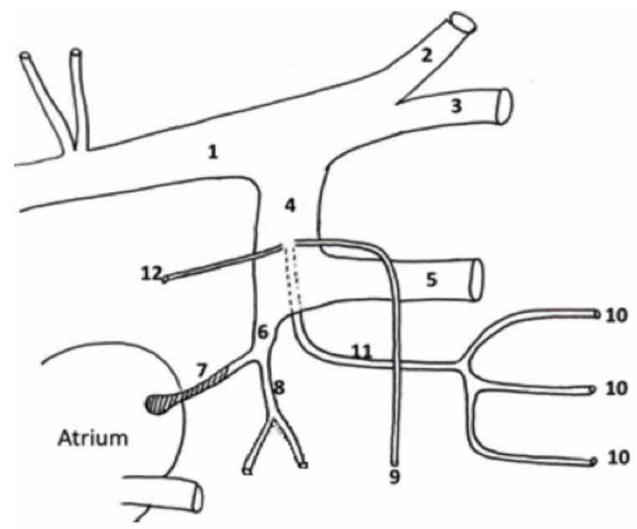

Fig. 4. Esquema de la conformación venosa anómala. 1. V. Braquiocefálica Izquierda; 2. V. Yugular Interna; 3. V. Subclavia; 4. Trayecto vertical anómalo de la V. Pulmonar Superior Izquierda; 5. V. Pulmonar Superior Izquierda (VPSI); 6. Confluencia venosa; 7. V. Pulmonar Superior Izquierda Obliterada; 8. V. Bronquial común; 9. V. Pericardiofrénica; 10. Vv. Intercostales; 11. V. Hemiácigos accesoria; 12. V. Pericárdica. Atrium = Atrio izquierdo. 


\section{DISCUSIÓN}

El desarrollo de la vascularización pulmonar ocurre tempranamente (día embrionario 27 al 37) y en paralelo con el establecimiento de los sistemas cardinal, vitelino y umbilical, compartiendo con estos sistemas conexiones temporales que normalmente desaparecen. La persistencia de una o más de estas conexiones embrionarias constituye la causa de aparición de anomalías de drenaje pulmonar que, consecuentemente, pueden involucrar conexiones al sistema cava, al sistema porta o a los atrios (Muñoz Castellanos et al., 2008). Durante el desarrollo pulmonar, la sangre procedente de los esbozos pulmonares drena al plexo esplácnico, el cual conecta a las venas cardiacas comunes con las umbilicovitelinas. Por su disposición y relaciones, la vena de trayecto anómalo que aquí presentamos tiene su origen en la persistencia de las conexiones del sistema venoso pulmonar como las descritas por Neill (1956), en especial las que se establecen con el sistema cardinal izquierdo y la vena vertical que no involuciona, (Muñoz Castellanos et al.); además, el drenaje venoso anómalo afecta solamente al lobo superior, tal como lo describe Brochet et al. (2014). La VPSI recibe en su trayecto el drenaje del pericardio, de ambas venas bronquiales izquierdas, de la vena pericardiofrénica izquierda, y de las venas intercostales posteriores izquierdas 3, 4 y 5 (Fig. 4). De modo tal que se mezcla sangre desoxigenada parietal y visceral del tórax con sangre oxigenada proveniente del lobo pulmonar superior izquierdo, la cual es vertida completamente a través de la VBCI hacia la circulación sistémica.

Una consecuencia fisiológica del drenaje anómalo de las venas pulmonares es una sobrecarga de volumen sobre las cámaras cardíacas derechas e hipervolemia en la circulación pulmonar. Las derivaciones totales requieren tratamiento quirúrgico, mientras que las derivaciones parciales que involucran a un sólo pulmón o lobo pulmonar, como en nuestro caso, se pueden dejar sin tratar controlando la evolución del paciente, cuyas manifestaciones clínicas dependerán del tamaño de la conexión anómala, de la presencia de un defecto septal atrial y del tiempo de desarrollo de la lesión (Podzolkov et al., 2016); sin embargo, la mayoría de los pacientes con conexiones venosas anómalas parciales son asintomáticos durante la infancia (Hijii et al.).

El caso que aquí presentamos corresponde a un drenaje pulmonar anómalo parcial, que determina que la VPSI drene hacia la VBCI (Fig. 1). La frecuencia de los sitios de conexión anómala en orden decreciente según Muñoz Castellanos et al. (2007) son: vena cava superior, seno coronario, atrio derecho y vena porta; en nuestro caso, la conexión se deriva hacia la VCS. La continuidad de la VPSI con la vena braquiocefálica izquierda y con la vena cava superior derecha conforma la imagen cardioangiográfica de la cabeza del "muñeco de nieve", mientras que el cuerpo lo forma la silueta cardíaca. Este tipo de anomalía se asocia generalmente con defecto en el tabique interatrial (Galletti \& Ramos Casado, 2010), tal como ocurre en el presente estudio, donde se detectó un foramen oval permeable (Fig. 3).

Las posibilidades de derivación hacia la circulación sistémica son variadas, Hijii et al., presentan una serie de 28 casos de drenajes pulmonares anómalos parciales, en los cuales las venas pulmonares derechas son las que presentan mayor variabilidad ( $89 \%)$ y pueden drenar en la vena cava superior, atrio derecho, vena cava inferior o en estas dos últimas a la vez, en tanto los drenajes pulmonares anómalos izquierdos son menos frecuentes $(7 \%)$ y pueden desembocar en al atrio derecho o la vena braquiocefálica izquierda, siendo esto concordante con lo planteado por Caputo et al. (2003) quien describe las anomalías de retorno venoso pulmonar izquierdo como una condición extremadamente rara. Bauer et al. (1982) presentan una serie de 7 casos con drenaje pulmonar anómalo del lado izquierdo recopilados en un período de 25 años (1955-1980), identificando a pacientes que van desde los 15 meses hasta los 40 años de edad. Otros autores como Alsoufi et al. (2007) estudiaron 306 casos en los cuales el $90 \%$ de los drenajes anómalos estaban asociados a lobos pulmonares del lado derecho, en tanto que Mascarenhas et al. (1973) describen que en 6 casos estudiados el $67 \%$ correspondió también al lado derecho. Haramati et al. (2003) presenta un estudio de 29 casos donde el $79 \%$ corresponde a drenaje anómalo de lobos pulmonares del lado izquierdo y por su parte Ho et al. (2009) realizaron un estudio de 47 pacientes donde el $47 \%$ corresponde a drenaje anómalo del lobo pulmonar superior izquierdo. Sin duda la presentación de estas anomalías asociadas a lateralidad es variable, sin embargo los estudios con muestra más amplia evidencian una predominancia derecha.

Las derivaciones parciales presentan pocas manifestaciones clínicas y se evidencian como un hallazgo clínico o durante disecciones, son más prevalentes en las venas pulmonares derechas y extremadamente raras en las venas pulmonares izquierdas. En nuestro trabajo presentamos los hallazgos de la disección de una anomalía venosa, que habitualmente se observa a través de imagen como angiografías, pero que se observa de manera menos frecuente en fotografías in situ.

Por último, el hallazgo de estas variaciones anatómicas en los cadáveres disecados con fines docentes en nuestro Departamento de Anatomía, tiene un valor formativo indiscutible para estudiantes de pregrado y postgrado, quienes pueden comprobar de primera mano la enorme variabilidad del 
ser humano, desterrando de sus mentes el arquetipo irreal y ficticio que presentan los modelos anatómicos, herramientas docentes de amplio uso hoy en día en las distintas escuelas de medicina (Inzunza, 2008; Inzunza et al., 2011).

\section{AGRADECIMIENTOS}

A las personas que en un loable acto de generosidad y grandeza donan su cuerpo a la ciencia para contribuir en la formación de mejores profesionales.

FARFÁN, C. E.; ECHEVERRÍA, M. M.; SALGADO, A. G.; NAVARRO, A. S.; INOSTROZA, R. V. \& INZUNZA, H. O. Anomalous pulmonary venous connection. A case report. Int. J. Morphol., 36(4):1197-1201, 2018.

SUMMARY: The anomalous connections of the pulmonary veins were first described in 1739. The drainage anomalies of the pulmonary veins are due to an early alteration (day 27-30 of development), during this period the pulmonary venous plexuses are in connection with the cardinal, umbilical and vitelline vein systems. These venous connections return and drain into the left atrium through the common pulmonary vein. In this work we present a partial anomalous pulmonary venous connection. Knowledge of this connection is relevant because it is associated to $80 \%$ of cardiac defects, mainly interatrial communications. During a routine dissection of an adult male cadaver, a vein originating in the left upper lobe was found. The vein originates at the pulmonary hilum in front of the bronchus, with an ascending path of a $10 \mathrm{~cm}$ length from the hilum, concluding its trajectory in the left brachiocephalic vein. The anomalous vein drains the left upper lobe of the lung. This anomalous vein originates within the connections of the pulmonary venous system, particularly those found in the left anterior cardinal vein system. This finding highlights the importance and clinical relevance of dissection in the training of health professionals.

KEY WORDS: Venous Connection; Anomalous Pulmonary Vein; Anatomical Variation.

\section{REFERENCIAS BIBLIOGRÁFICAS}

Alsoufi, B.; Cai, S.; Van Arsdell, G. S.; Williams, W. G.; Caldarone, C. A. $\&$ Coles, J. G. Outcomes after surgical treatment of children with partial anomalous pulmonary venous connection. Ann. Thorac. Surg., 84(6):2020-6, 2007.

Bauer, A.; Körfer, R. \& Bircks, W. Left-to-right shunt at atrial level due to anomalous venous connection of left lung: report of seven cases. $J$. Thorac. Cardiovasc. Surg., 84(4):626-30, 1982.

Brochet, G.; Giraldo Vallejo, F.; Manrique, J.; López, F.; Fernández, O.; Manrique, F. \& Sarmiento, P. Drenaje venoso pulmonar anómalo parcial del pulmón izquierdo. Corrección mediante toracotomía sin circulación extracorpórea. Rev. Colomb. Cardiol., 21(1):68-71, 2014.
Caputo, M.; Carcagni, A.; Presbitero, P. \& Gallotti, R. Partial anomalous left pulmonary venous return. J. Cardiovasc. Surg. (Torino), 44(1):613, 2003.

Galletti, L. \& Ramos Casado, M. Anomalías de las Venas Pulmonares. En: Zabala Argüelles, J. I. (Ed.). Protocolos Diagnósticos y Terapéuticos en Cardiología Pediátrica. Madrid, Sociedad Española de Cardiología Pediátrica y Cardiopatías Congénitas, 2010.

Haramati, L. B.; Moche, I. E.; Rivera, V. T.; Patel, P. V.; Heyneman, L.; McAdams, H. P.; Issenberg, H. J. \& White, C. S. Computed tomography of partial anomalous pulmonary venous connection in adults. J. Comput. Assist. Tomogr., 27(5):743-9, 2003.

Hijii, T.; Fukushige, J. \& Hara, T. Diagnosis and management of partial anomalous pulmonary venous connection. A review of 28 pediatric cases. Cardiology, 89(2):148-51, 1998.

Ho, M. L.; Bhalla, S.; Bierhals, A. \& Gutierrez, F. MDCT of partial anomalous pulmonary venous return (PAPVR) in adults. J. Thorac. Imaging, 24(2):89-95, 2009.

Inzunza, A. M.; Salgado, A. G.; González, S. A.; De la Cuadra, J. C. \& Inzunza, H. O. Massive communication between the superficial branch of radial nerve and lateral cutaneous nerve of the forearm, anatomical and clinical implications. A case report. Int. J. Morphol., 29(3):681-5, 2011.

Inzunza, H. O. Competencias generales en medicina, rol de la Anatomía. Int. J. Morphol., 26(2):243-6, 2008.

Magalhães, S. P.; Moreno, N.; Loureiro, M.; França, M.; Reis, F.; Alvares, S. \& Ribeiro, M. Anomalous pulmonary venous connection: An underestimated entity. Rev. Port. Cardiol., 35(12):697.e1-697.e6, 2016.

Mascarenhas, E.; Javier, R. P. \& Samet, P. Partial anomalous pulmonary venous connection and drainage. Am. J. Cardiol., 31(4):512-8, 1973.

Muñoz Castellanos, L.; Kuri Nivón, M.; Sánchez Vargas, C. A. \& Espínola Zavaleta, N. Conexión anómala de venas pulmonares. Correlación anatomoembriológica. Arch. Cardiol. Mex., 78(3):247-54, 2008.

Muñoz Castellanos, L.; Sánchez Vargas, C. A. \& Kuri Nivon, M. Estudio morfopatológico de la conexión anómala total de venas pulmonares. Arch. Cardiol. Mex., 77(4):265-74, 2007.

Neill, C. A. Development of the pulmonary veins; with reference to the embryology of anomalies of pulmonary venous return. Pediatrics, 18(6):880-7, 1956.

Orts Llorca, F. Anatomía Humana. Tomo Tercero. Barcelona, Científico Médica, 1972.

Podzolkov, V. P.; Chiaureli, M. R.; Kovalev, D. V. \& Zaets, S. B. Repair of isolated partial anomalous pulmonary venous connection of the right upper pulmonary veins to the left vertical vein. Interact. Cardiovasc. Thorac. Surg., 23(3):501-2, 2016.

Sadler, T. W. Langman's Medical Embryology. $13^{\text {th }}$ ed. Philadelphia, Lippincott Williams \& Wilkins, 2014.

Schoenwolf, G.; Bleyl, S.; Brauer, P. \& Francis-West, P. Larsen's Human Embriology. $4^{\text {th }}$ ed. Philadelphia, Elsevier Churchill Livingstone, 2009.

\section{Dirección para correspondencia \\ Emilio Farfán C. \\ Departamento de Anatomía \\ Escuela de Medicina \\ Pontificia Universidad Católica de Chile \\ Santiago \\ CHILE}

Email: efarfanc@uc.cl

Recibido : 25-01-2018

Aceptado: 24-07-2018 